\title{
A Novel Pilot Expansion Approach for MIMO Channel Estimation and Tracking
}

\author{
MingFei Siyau, \\ School of Engineering \& Design \\ London South Bank University \\ London, UK \\ siyaum@1sbu.ac.uk
}

\author{
Tiancheng Li, Javier Prieto, Juan Corchado \\ School of Science \\ University of Salamanca \\ Salamanca, Spain \\ \{t.c.li, javierp, corchado\} usal.es
}

\author{
Javier Bajo \\ Dept. of Artificial Intelligence \\ Technical University of Madrid \\ Madrid, Spain \\ jbajo@fi.upm.es
}

\begin{abstract}
Enabling high practical mobility of MIMO-OFDM systems requires accurate channel estimation under severe frequency and time selective fading channels. Conventional methods rely on non-realistic approximations or result in impractical complexities. In this paper, a new practical trainingbased MIMO channel estimation scheme is presented. The new pilot bits designed from the 'Paley-Hadamard' matrix allows exploiting its orthogonal and 'Toeplitz-like' structures for minimising its pilot length. Additionally, a novel pilot expansion technique is proposed to estimate the length of the channel impulse response, by flexibly extending its pilot length as required to capture the number of multipath existed within the MIMO channel. The proposed pilot expansion enables to deduce the initial channel variation and its Doppler rate, which can be subsequently applied for MIMO channel tracking using decision feedback Kalman filter during the data payload.
\end{abstract}

Index Terms- MIMO channel estimation, Pilot Expansion technique, Kalman tracking.

\section{INTRODUCTION}

The multiple-input multiple-output (MIMO) system has been recognized as one of the key solutions for future generation wireless communication e.g. wireless highways [1] due to its capability to exploit multipath while realizing high performance in spatial multiplexing, array and power gain. Merging with existing orthogonal frequency division multiplexing (OFDM) technologies, MIMO-OFDM systems can boost data transmission rate while handling frequencyselective (FS) fading [2-3]. However, to allow and enable high mobility practicability, such systems must also be capable to obtain accurate channel information for symbol recovery in high speed while tackling time selective (TS) fading and cochannel interference (CCI).

Various smart channel estimation (CE) [4-7, 12-15] and channel tracking (CT) techniques [8-11] have been proposed to provide estimates on the channel impulse response (CIR) or channel frequency response (CFR). Many researches focus on efficient pilot design and allocation methods $[6,7,12,13,21$ 26] for separate FS and TS cases where [13, 25] give excellent overview on pilot requirements. In general, the trend of assuming or relying on the prior knowledge of a) the length of CIR and b) Doppler rate, frequently become the bottleneck to perform well in real TS and FS fading channels, especially in wireless highways. Many methods build on approximation and rely on sufficiently high-order polynomial model resulting in complex algorithms $[8,9,12,14,15]$, rendering impractical solution for real time $\mathrm{CE}$ where the optimality of the pilot ultimately depends on the speed of CE.

In this paper, both MIMO-CE and CT schemes have been devised to realise practical communications in both FS and TS channels. First, pilot bits are carefully designed to shorten the length of the training period via a unique combination of the 'orthogonal' and 'Toeplitz-like' structure ascertained from the integrated Paley-Hadamard (P-H) matrix [16, 17]; extension to a fractionally-sampled case can be found in [20] as an earlier version of this paper. The latter facilitates the resolution of both $\mathrm{CCI}$ and inter-symbol interference (ISI) while estimating individual CIR on each transmit $(T x)$ and receive $(R x)$ antenna pairs. The novelty of this paper is the next 'Pilot Expansion' (PE) technique which has the capability to further exploit the P$\mathrm{H}$ code structure and to flexibly extend the pilot length as required, in order to a) approximate the length of CIR, $L$ and b) reveal the time-variation information of the respective TS channels. The proposed PE technique is also crucial for the subsequent provision of channel tracking for individual CIR or CFR to ensure receiver's resiliency against severe TS fading environment and achieve accurate receiver's setting during the symbol recovery and decision feedback process. Here, the MIMO-OFDM system is chosen to showcase the proposed CE, $\mathrm{PE}$ and CT methods, while their extensions to any future MIMO-based communication systems is straightforward.

The rest of the paper is organized as follows. Section-II describes the basic channel model. Section-III presents the MIMO-CE and a novel pilot expansion technique. Section-IV presents Kalman-CT. Section-VI shows the simulation results and discussion. Finally, section-VI concludes the paper.

Notations: $(.)^{\mathrm{T}}$ is denoted for the transpose operation and $(.)^{\mathrm{H}}$ is denoted for the Hermitian operation.

\section{MIMO CHANNEL MODEL}

In this paper, severe TS and FS fading channels have been assumed and simulated using the Jakes and Bello models [1819]. The time domain (TD) time-varying sampled CIR from $i^{\text {th }}-T x$ antenna to $j^{\text {th }}-R x$ antenna can be termed as:

$$
\mathbf{h}_{j i}(t)=\left[\begin{array}{llll}
h_{j i}(0, t) & h_{j i}(1, t) \quad \ldots & h_{j i}(L-1, t)
\end{array}\right]^{\top}
$$


where $L$ is number of multipath on each $T x$ - $R x$ antenna pair. $h_{j i}(l, t)$ is the individual TD channel coefficient on $l^{\text {th }}$ path at time $t$. The MIMO channel will be formed by $\mathbf{h}_{j i}(t)$ shown in (4) later. The corresponding time-varying sampled CFR from $i^{\text {th }} T x$ to $j^{\text {th }} R x$ antenna can be found by applying the inverse fast-Fourier transform (ifft) function to $\mathbf{h}_{j i}(t)$ in (1) as:

$$
\mathbf{g}_{j i}(t)=i f f t\left(\mathbf{h}_{j i}(t), N\right)=\left[\begin{array}{llll}
g_{j i}(0, t) & g_{j i}(1, t) & \ldots & g_{j i}(N-1, t)
\end{array}\right]^{\top}
$$

where $N$ is the number of ifft point $g_{j i}(n, t)$ is the individual frequency domain (FD) channel coefficient on $n^{\text {th }}$ subcarrier at time $t$. The time-correlation between the adjacent TD coefficients is governed by the Doppler rate $f_{D} T,\left(f_{D}\right.$ is the maximum Doppler frequency and $T$ is the baud duration) as:

$$
E\left\{h_{j i}\left(t_{1}\right) h_{j i}^{*}\left(t_{2}\right)\right\} \approx J_{0}\left(2 \pi f_{D} T\left|t_{1}-t_{2}\right|\right)
$$

where $J_{0}(\cdot)$ is the zero-order Bessel function of the first kind. Thus, the resultant $g_{j i}(n, t)$ in (2) must have the same timevarying property with the similar Doppler rate and the timecorrelation between the adjacent FD coefficients as $h_{j i}(l, t)$.

\section{MIMO CHANNEL ESTIMATION SCHEME}

A MIMO-CE scheme in Fig.-1 is proposed to concurrently estimate corresponding CIRs and its ' $\mathrm{L}$ ' length while revealing the channel variation information via a novel pilot expansion (PE) technique utilizing flexible PE models shown in (12) and (13) later that 'encompass' the FS MIMO channel for TS case.

\section{A. MIMO-CE utilizing Paley-Hadamard codes}

To provide a virtual 'division' on individual CIR in FS case, each basic channel matrix consisting of all CIRs, $\mathbf{h}_{j i}(t)$, with respect to (w.r.t) each $T x$ antenna can be written as:

$$
\mathbf{U}_{i}(t)=\left[\begin{array}{c}
\mathbf{h}_{1 i}^{\top}(t) \\
\vdots \\
\mathbf{h}_{N_{r} i}^{\top}(t)
\end{array}\right]=\left[\begin{array}{ccc}
h_{1 i}(0, t) & \cdots & h_{1 i}(L-1, t) \\
\vdots & \ddots & \vdots \\
h_{N, i}(0, t) & \cdots & h_{N_{,} i}(L-1, t)
\end{array}\right]
$$

where ' $i$ ' $=1$ to $N_{\mathrm{t}}$ and $L$ multipath exists in each CIR. Next, to facilitate an efficient MIMO-CE, all $\mathbf{U}_{i}(t)\left(i=1, \ldots, N_{\mathrm{t}}\right)$ can be concatenated to form the complete MIMO channel as:

$$
\mathbf{U}_{\text {all }}(t)=\left[\begin{array}{lll}
\mathbf{U}_{1}(t) & \cdots & \mathbf{U}_{N_{t}}(t)
\end{array}\right]
$$

The time-index ' $t$ ' is temporarily changed to ' $n$ ' in order to differentiate the 'virtual variation' due to ' $n$ ' sequence of pilot bits as oppose to the actual variation in time. Thus, TD signal interactions at $n^{\text {th }}$ sample at the receiver can be viewed as:

$$
\begin{gathered}
\mathbf{y}(n)=\mathbf{U}_{\text {all }}(n) \mathfrak{I}(n)=\left[\begin{array}{lll}
y_{1}(n) \cdots y_{N_{r}}(n)
\end{array}\right]^{\top} \\
\mathfrak{I}(n)=\left[\begin{array}{llll}
\mathbf{s}_{1}(n) & \cdots & \mathbf{s}_{N_{t}}(n)
\end{array}\right]^{\top} \\
\mathbf{s}_{i}(n)=\left[\begin{array}{llll}
s_{i}(n) & s_{i}(n-1) & \cdots & s_{i}(n-L+1)
\end{array}\right]^{\top}
\end{gathered}
$$

Note that $\mathfrak{I}(n)$ consists of $N_{t}$ number of $T x$ vector, $\mathbf{s}_{i}(n)$, sent by individual $i^{\text {th }} T x$ antenna. Each $\mathbf{s}_{i}(n)$ contains $L$ length of $T x$ pilot bits; from current $n^{\text {th }}$ bit to past $(n-L+1)^{\text {th }}$ bit, which convolve with different coefficients $h_{j i}(n, t)$. The aim is to design pilot codes in $\mathbf{s}_{i}(n)$ such that pilot vectors $\mathfrak{I}(n)$ can differentiate individual $T x$ antenna for virtual segregation of all its CIRs. The pilot matrix, $\mathbf{S}_{\text {pilot }}$ can be formed as:

$$
\mathbf{S}_{\text {pilot }}=\left[\begin{array}{llll}
\mathfrak{I}(n) & \mathfrak{I}(n+1) & \cdots & \mathfrak{I}(n+v-1)
\end{array}\right]
$$

where ' $v$ ' is the chosen matrix order. Each row sequence in $\mathbf{S}_{\text {pilot }}$ is designed to be orthogonal w.r.t other row sequences. Next, the CE can be performed with ' $L$ ' known a priory as:

$$
\hat{\mathbf{U}}_{\text {all }}=\mathbf{Y ~ \mathbf { S } _ { \text { pilot } } ^ { \mathrm { H } }}
$$

where $\mathbf{Y}$ is the corresponding $R x$ pilot matrix with the proper samples collection of $\boldsymbol{y}(n)$ at every ' $L$ ' intervals as:

$$
\mathbf{Y}=\left[\begin{array}{llll}
\mathbf{y}(n) & \mathbf{y}(n+L) & \cdots & \mathbf{y}(n+(v-1) L)
\end{array}\right](11)
$$

The challenge is to design $\mathbf{S}_{\text {pilot }}$ such that the gap between the collection intervals of $\boldsymbol{y}(n)$ in $\mathbf{Y}$ can be further reduced to include adjacent samples, while ensuring the CE accuracy in (10). This can be achieved by utilizing the P-H matrix codes which provides both orthogonal and 'Toeplitz-like' unique combined structure. An example of pilot matrix with the P-H codes for $v=8, N_{t}=2 L \leq 4$ is shown below:

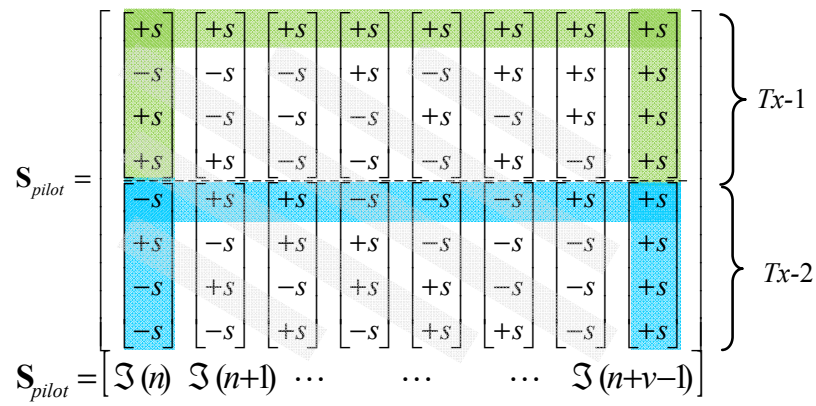

A thorough 'run-through' from (4) to (11), reveals that the diagonally Toeplitz-like structure can be exploited such that only $v+2(L-1)$ number of pilot bits (instead of $v L$ ) are required per $T x$ antenna, as shown in the colored regions. Pilot bits in those non-colored regions can avoid to be sent, while retaining the same $\mathbf{S}_{\text {pilot }}$ formation at the receiver for CE in (10). This allows almost every successive samples of $\boldsymbol{y}(n)$ to be utilized instantly without gap, instead of waiting every ' $L$ ' interval as in (11), thereby further shortening the pilot length and overall CE processing time that is crucial in the TS channels case.

\section{B. Novel Pilot Expansion (PE) Technique}

Next, the MIMO-CE scheme is further developed to a) estimate the length of CIRs and b) reveal channel variation. In reality, length ' $L$ ' in each CIR is unknown, while the ultimate size of $\widehat{\mathbf{U}}_{\text {all }}$ in (10) depends solely on the amount of samples collection $\boldsymbol{y}(n)$ in $\mathbf{Y}$ and corresponding $\mathbf{S}_{\text {pilot }}$ formation at the receiver. Thus, it is vital to allow flexible extension in pilot length to cater every possible ' $L$ ' while keeping the MIMOCE process intact. To effectively 'capture' this information, an approximation PE model of $\mathbf{U}_{i}(t)$ in (4) can be formed as:

$$
\breve{\mathbf{U}}_{i}(t)=\left[\begin{array}{c}
\breve{\mathbf{h}}_{1 i}^{\top}(t) \\
\vdots \\
\breve{\mathbf{h}}_{N_{r} i}^{\top}(t)
\end{array}\right]=\left[\begin{array}{ccc}
\breve{h}_{1 i}(0, t) & \cdots & \breve{h}_{1 i}\left(L_{\text {est }}-1, t\right) \\
\vdots & \ddots & \vdots \\
\breve{h}_{N, i}(0, t) & \cdots & \breve{h}_{N, i}\left(L_{\text {est }}-1, t\right)
\end{array}\right]
$$

where $L_{\text {est }}$ is the 'guess' length of CIR and $L_{e s t} \geq L$ so that estimated $\breve{\mathbf{U}}_{i}(t)$ in (12) is always wider than $\mathbf{U}_{i}(t)$ in (4). Intuitively, sending a longer pilot sequence of pilot vectors will ensure that the estimate of $\breve{\mathbf{h}}_{j i}^{\mathrm{T}}(t)$ is always sufficient to 


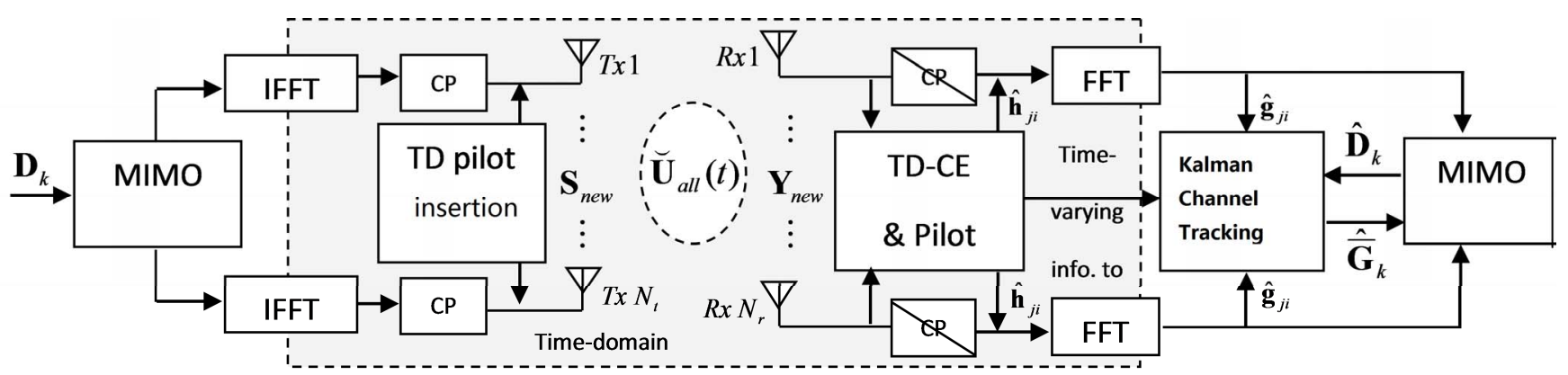

Fig.1: Proposed Channel Estimation with novel Pilot Expansion Scheme and Kalman Channel Tracking

approximate the actual $\mathbf{h}_{j i}(t)$, with additional $\left(L_{e s t}-L\right)$ CIR 'residues' at the tail of $\mathbf{h}_{j i}^{\mathrm{T}}(t)$ estimate. These residues are usually near 'zero' value, which can be removed (or further utilized). However, using only a single $\breve{\mathbf{U}}_{i}(t)$ PE model in (12) can render insufficient $\mathrm{CE}$ accuracy in the high noise case. Thus, to guarantee a better expectation of $\mathrm{CE}$, a further extended approximation PE model on $\mathbf{U}_{\text {all }}(t)$ is required as:

$$
\breve{\mathbf{U}}_{\text {all }}(t)=\left[\begin{array}{cccccc}
\breve{\mathbf{U}}_{1}(t) & \cdots & \breve{\mathbf{U}}_{N_{t}}(t) & 0 & \cdots & 0 \\
\ddots & & \ddots & & \\
0 \cdots 0 & \breve{\mathbf{U}}_{1}(t+M) & \cdots & \breve{\mathbf{U}}_{N_{t}}(t+M)
\end{array}\right]
$$

where $\breve{\mathbf{U}}_{\text {all }}(t)$ contains $(M+1)$ blocks of $\breve{\mathbf{U}}_{i}(t)$ in succession which implies they can now be jointly evaluated to increase the $\mathrm{CE}$ accuracy. For a time-invariant channel, all $\breve{\mathbf{U}}_{i}(t)$ to $\breve{\mathbf{U}}_{i}(t+$ $M)$ will be quite similar, thus, render a positive estimation of $\breve{\mathbf{U}}_{i}(t)$. When the channel is time-varying, changes in CIRs are also gradually revealed across from all $\breve{\mathbf{U}}_{i}(t)$ to $\breve{\mathbf{U}}_{i}(t+M)$, providing the vital clue to unveil the time-variation information (i.e. the Doppler rates and travel velocities) while the basic $\mathrm{CE}$ is carried out. In fact, $L_{\text {est }}$ can initially take a larger value to include $M$ in order to estimate $\breve{\mathbf{U}}_{i}(t)$ and the length of CIR in (12) first (as the resultant residues will implicate the actual CIR length) and then adapt $M$ as $\left(L_{e s t}-L\right)$ to further process $\breve{\mathbf{U}}_{\text {all }}(t)$ in (13).

To achieve this, $\boldsymbol{S}_{\text {pilot }}$ in (9) must be capable to be flexibly expanded to satisfy the formation of $\breve{\mathbf{U}}_{\text {all }}(t)$ in (13), while keeping pilot length to minimum requirement and resolving CCI, FS and TS channels concurrently for a large MIMO channel during $\mathrm{CE}$, which is the key highlight in the PE technique. To show this, pilot bits in $\mathbf{s}_{i}(n)$ in $(8)$ are extended to include $\left(M+L_{\text {est }}-L\right)$ additional bit per $T x$ antenna as:

$$
\breve{\mathbf{s}}_{i}(n)=\left[\begin{array}{llll}
s_{i}(n) & s_{i}(n-1) & \cdots & s_{i}\left(n-L_{e s t}-M-1\right)
\end{array}\right]^{\top}
$$

This will render a corresponding $\mathfrak{\Im}_{\text {new }}(n)$ and $\mathbf{B}(n)$ as::

$$
\mathbf{B}(n)=\breve{\mathbf{U}}_{\text {all }}(n) \mathfrak{I}_{\text {new }}(n)=[\mathbf{y}(n+M) \cdots \mathbf{y}(n)]^{\top}
$$

where $\mathbf{B}(n)$ now contains several basic vector $\boldsymbol{y}(n)$ in (6) in succession. $\Im_{\text {new }}(n)$ can still be formulated using (7) with $\breve{\boldsymbol{s}}_{i}(n)$ in (14) replacing $\boldsymbol{s}_{i}(n)$ in (8). Thus, the new pilot matrix $\mathbf{S}_{\text {new }}$ expanded from (9) with a new Hadamard order- $v$ :

$$
\mathrm{v}>N_{t} \times\left(L_{e s t}+M\right)
$$

Hence, the new $\mathrm{CE}$ with pilot expansion requires total pilot bits of $v+2\left(L_{e s t}+M-1\right)$ per $T x$ antenna and is performed as:

$$
\hat{\mathbf{U}}_{\text {all }}=\mathbf{Y}_{\text {new }} \mathbf{S}_{\text {new }}^{\mathrm{H}}
$$

where $\mathbf{Y}_{\text {new }}$ is the corresponding new $R x$ pilot matrix with the proper blocks collection of $\mathbf{B}(n)$ from (15) as:

$$
\mathbf{Y}_{\text {new }}=\left[\begin{array}{lll}
\mathbf{B}(n) \mathbf{B}(n+1) \cdots \mathbf{B}(n+v-2) & \mathbf{B}\left(n+v+L_{e s t}+M-1\right)
\end{array}\right]
$$

Note that there is a short waiting gap between the last two blocks $\mathbf{B}(n+v-2)$ and $\mathbf{B}\left(n+v+L_{e s t}+M-1\right)$ in order to obey the crucial P-H formation in $\boldsymbol{S}_{\text {new }}$ needed in (17). Once $\widehat{\mathbf{U}}_{\text {all }}(t)$ is obtained, each $\breve{\mathbf{U}}_{i}(t)$ within can be jointly evaluated for the channel time-variation information and its ' $L$ ' length in CIRs.

\section{KALMAN CHANNEL TRACKING}

To complement the proposed PE method in TS channel case, the Kalman approach [10] is used for channel tracking. $\mathrm{TD} \hat{\mathbf{h}}_{j i}(t)$ are changed into $\mathrm{FD} \hat{\mathbf{g}}_{j i}(t)$ and re-arranged as $\overline{\mathbf{G}}_{k}$, and tracked subsequently by the Kalman algorithm below:

Step 1. Predicting Equations

$$
\begin{aligned}
& \hat{\overline{\mathbf{G}}}_{k+1}^{-{ }^{\top}}=\mathbf{A} \hat{\overline{\mathbf{G}}}_{k}{ }^{\top} \\
& \mathbf{P}_{k+1}^{-{ }^{\top}}=\mathbf{A} \mathbf{P}_{k} \mathbf{A}^{\top}+Q
\end{aligned}
$$

Step 2. Measurement Equations

$$
\begin{aligned}
& \mathbf{K}_{k}=\mathbf{P}_{k}^{-}\left(\hat{\mathbf{D}}_{k}^{\top}\right)^{\mathrm{H}}\left(\hat{\mathbf{D}}_{k}^{\top} \mathbf{P}_{k}^{-}\left(\hat{\mathbf{D}}_{k}^{\mathrm{T}}\right)^{\mathrm{H}}+\sigma^{2}\right)^{-1} \\
& \hat{\mathbf{G}}_{k}^{\top}=\hat{\mathbf{G}}_{k}^{-\top}+\mathbf{K}_{k}\left(\mathbf{X}_{k}^{\top}-\hat{\mathbf{D}}_{k}^{\top} \hat{\mathbf{G}}_{k}^{-\top}\right) \\
& \mathbf{P}_{k}=\left(\mathbf{I}_{N_{t} N}-\mathbf{K}_{k} \hat{\mathbf{D}}_{k}^{\top}\right) \mathbf{P}_{k}^{-}
\end{aligned}
$$

where $\mathbf{K}_{k}$ is the Kalman gain. $\mathbf{P}_{k}$ and $\mathbf{P}_{\boldsymbol{k}}^{-}$are the posterior and prior estimate error covariance matrices, respectively, $\mathbf{I}_{N_{t} N}$ is the $N_{t} N$-size identity matrix, $Q$ and $\sigma^{2}$ are noise covariance for the process and measurement models. $\mathbf{A}$ is the channel transition matrix. All initialization parameters above are properly tuned to yield a good CT mechanism, refer to [10] therein for further insight.

As mentioned in (3), the FD coefficients in CFRs have the same time-variation property as the TD channel coefficients in CIRs, where the time-correlation between the adjacent samples follow similar Doppler rate, $f_{d} T$ in Bello model [19]. Hence, it is vital to reflect this information in $\mathbf{A}$ in order to track ahead the estimates accurately. The estimate of $\breve{\mathbf{U}}_{\text {all }}(t)$ offers some vital clues as the displacements of coefficients from $\widetilde{\mathbf{U}}_{i}(t)$ to $\breve{\mathbf{U}}_{i}(t+M)$ in (13) and can be used 
to predict the corresponding mobile velocity, $\hat{\mu}$ and the Doppler shift frequency, $\hat{f}_{d}$ (from the known $T x$ bit rate, $T$ ). With these estimates, the variation pattern of each coefficient in CFR can be approximated: First, forming a virtual waveform as: $\cos \left(2 \pi \hat{f}_{d} T+\theta_{j i}(l)\right)$ with the phasor $\theta_{j i}(l)$ defined for each ' $l$ ' path from $i^{\text {th }} T x$ to $j^{\text {th }} R x$ antenna. Next, taking the difference between every adjacent sample-pair and normalize them. The result offers a weighted projection 'gradient' with the variant rate similar to (3) and can be combined with the actual variation magnitude of each CFR coefficient that has already been revealed in the changes from $\breve{\mathbf{U}}_{i}(t)$ to $\breve{\mathbf{U}}_{i}(t+M)$ in (13) for time variation on the channels.

\section{SiMULATIONS}

The results of the proposed MIMO-CE with the novel PE technique are studied using the parameters set in Table 1 below and the mean Frobenius norm squared error (MSE) of the channel estimates as compared to the original channels. For consistency, the MSEs are taken at the mid-length of the total pilot bits sent per each $T x$ antenna. The QPSK modulation scheme is used throughout the simulation model.

\begin{tabular}{|c|c|c|}
\multicolumn{3}{c}{ Table-I: Parameters for Simulation } \\
\hline Symbol & Parameter & Value \\
\hline$f_{c}$ & Carrier frequency & $2.1 \mathrm{GHz}$ \\
\hline$R$ & Data rate per $T x$ & $600 \mathrm{kbps}$ \\
\hline$\mu$ & Vehicle mobile velocity & $55 \mathrm{~km} / \mathrm{hr}, 115 \mathrm{~km} / \mathrm{hr}$ \\
\hline$L$ & Length of CIR & 3 (equally spaced by one sample) \\
\hline$N$ & FFT/IFFT size & 512 \\
\hline$C$ & Frame length & 200 OFDM symbols \\
\hline$N_{r} \times N_{t}$ & MIMO configuration & $(3 \times 2): 2 T x \& 3 R x$ antenna \\
\hline
\end{tabular}

Fig. 2 shows the impact of the chosen ' $M$ ' values of the PE technique on the estimation accuracy at different mean SNRs for a $\mu=55 \mathrm{~km} / \mathrm{hr}$ channel where the MSE improves as ' $M$ ' increases for better averaging of channel estimates. The results suggested that using ' $M$ ' blocks of $\breve{\mathbf{U}}_{i}(t)$ for joint evaluation, can help to further improve the channel estimates. However, as shown in Fig.3 later as ' $M$ ' becomes higher, the MSEs tends to depreciate due to the variation of the channel interaction with individual pilot bits. Fig. 3 compares the case using two channel velocities at $\mathrm{SNR}=15 \mathrm{~dB}$ and shows the impact of having PE technique as the pilot length increases. For $(M=0)$ case, where pilot length is allowed to increase without the help of PE, the result seems to suggest that there is an optimal pilot length for different channel speeds that limits the estimates to be further improved. With the use of PE ( $M$ increment), the $\mathrm{CE}$ can cope better with the channel variations as MSEs have been improved as compared to the case without the use of PE technique. Fig. 3 is based on $L_{e s t}=L=3$. For CE with the PE assistance, the total pilot length: $v+2\left(L_{e s t}+M-1\right)$ required per $T x$ antenna (as the valid matrix order- $v$ in (16) and block ' $M$ ' of PE increases) is given in Table II. There is an optimal pilot length for different channel speeds that limits the estimates to be further improved.

Table-II: Parameters for Simulation

\begin{tabular}{|c|c|c|c|c|c|c|}
\hline ' $M$ ' block of pilot expansion & 0 & 2 & 4 & 6 & 8 & 10 \\
\hline Pilot matrix order ' $v$ ' & 8 & 12 & 20 & 20 & 24 & 32 \\
\hline Pilot bits sent per $T x$ antenna & 12 & 20 & 32 & 36 & 44 & 56 \\
\hline
\end{tabular}

Next, the behavior of the CE and CT is jointly studied where CE pilot training is carried out for every data frame followed by the Kalman-CT for each OFDM symbol delivery within the frame. The bit error rate (BER) system performance of a (3×2) MIMO-OFDM system under both TS and FS fading channels is shown in Fig.4. The results are plotted for two mobile velocities for three different conditions: a) perfect $\mathrm{CE}$, b) $\mathrm{CE}$ with $\mathrm{PE} \&$ Kalman-CT and c) $\mathrm{CE}$ without $\mathrm{PE}$ \& Kalman-CT. The results show the potential of the proposed CE-PE and Kalman-CT features in achieving a better symbol recovery and BER when utilized together, outperforming conventional $\mathrm{CE}$ and $\mathrm{CT}$.

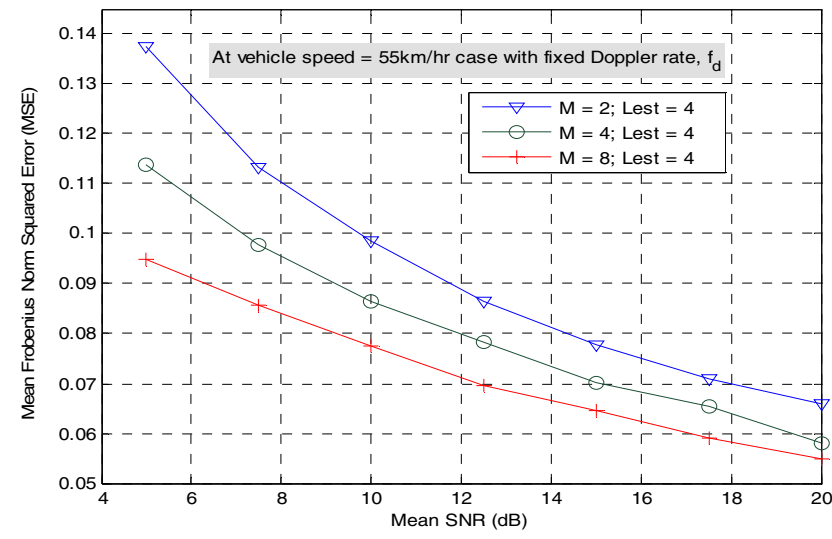

Fig.-2. The performance of PE with different $M$, for $55 \mathrm{~km} / \mathrm{hr}$ channel.

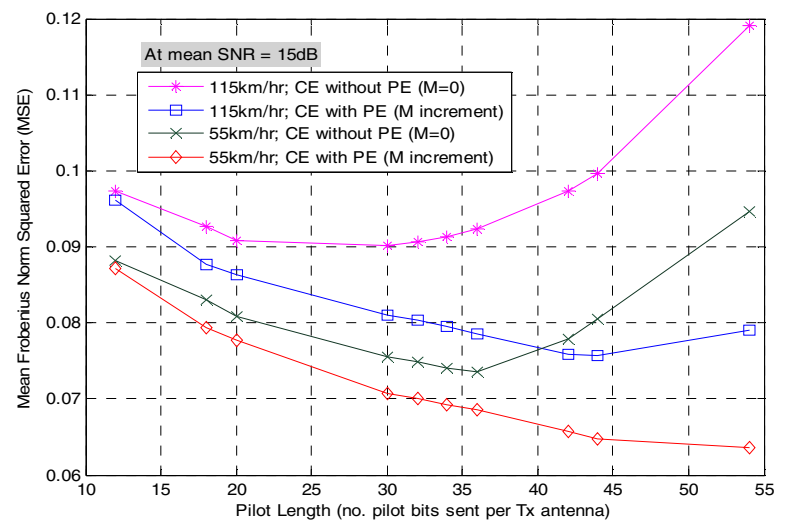

Fig.-3. Impact of CE with/without PE for $\mu=55 \mathrm{~km} / \mathrm{hr} \& 115 \mathrm{~km} / \mathrm{hr}$ channels.

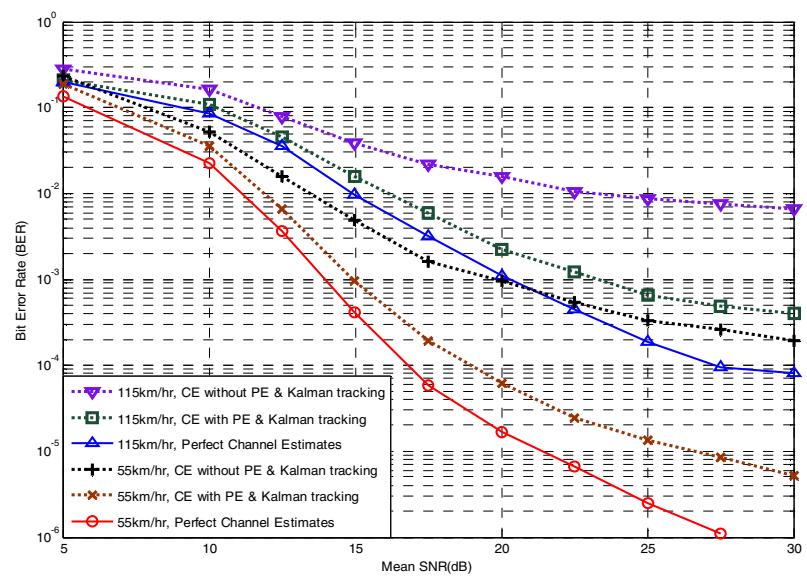

Fig. 4. The BER performance of $(3 \times 2)$ MIMO-OFDM system with $\mu=55 \mathrm{~km} / \mathrm{hr} \& 115 \mathrm{~km} / \mathrm{hr}$, and $R=600 \mathrm{kbps} / T x$. 


\section{CONCLUSION}

This paper has presented a MIMO channel estimator with a new Pilot Expansion (PE) technique for MIMO-based systems that operate in both frequency and time selective fading channel. The advantage of having the PE technique is that the new jointly CE-PE scheme can effectively predict the length of CIR and channel time-variation as well as carry out more robust channel estimation for MIMO systems. The Kalman channel tracking algorithm with decision feedback has also been incorporated to assist the CE-PE process and cater for TS channels case. The performance of the joint CE \& CT has been corroborated and it is found that the proposed method is capable to provide effective MIMO channel estimation with potential tracking provision. The proposed PE methods yield a positive BER performance that is close to the results when perfect channel estimates and linear tracking are assumed.

\section{ACKNOWLEDGMENT}

This work is partly supported by the project Sociedades Humano-Agente en entornos Cloud Computing (Soha+C). Project co-financed with Junta Castilla y León funds.

\section{REFERENCES}

[1] D. Benyamina, A. Hafid, M. Gendreau, "Wireless Mesh Networks Design - A Survey," Communications Surveys \& Tutorials, IEEE, vol.14, no.2, pp.299-310, 2012.

[2] G. L. Stuber, J. R. Barry, S. W. McLaughlin, Y. Li, M. A. Ingram, T.G. Pratt, "Broadband MIMO-OFDM Wireless Communications," Proc. of IEEE, vol. 92, no. 2, pp. 271-294, 2004.

[3] Y.G. Li, J.H. Winters, N.R. Sollenberger, "MIMO-OFDM for wireless communications: signal detection with enhanced channel estimation," IEEE Trans. Commun., vol. 50, no. 9, pp. 1471-1477, Sept. 2002.

[4] H. Yin, D. Gesbert, M. Filippou, Y. Liu, A Coordinated Approach to Channel Estimation in Large-scale Multipleantenna Systems, arXiv:1203.5924, 2012.

[5] D. Angelosante, E. Biglieri, M. Lops, "Sequential Estimation of Multipath MIMO-OFDM Channels," IEEE Trans. On Sign. Proc., vol. 57, no. 8, pp. 3167-3181, 2009.

[6] Q. Huang, M. Ghogho, S. Freear, "Pilot Design for MIMO OFDM Systems With Virtual Carriers," IEEE Trans. Signal Processing, vol. 57, no. 5, pp. 2024-2029, May. 2009.

[7] I. Barhumi, G. Leus M. Moonen, "Optimal training design for MIMO OFDM systems in mobile wireless channels," IEEE Trans. on Sign. Proc., vol. 51, no. 6, pp. 1615-1624, 2003.

[8] W.-G. Song J.-T. Lim, "Channel estimation and signal detection for MIMO-OFDM with time varying channels," IEEE Commun. Letter, vol. 10, no. 7, pp. 540-542, July. 2006.

[9] A. Duel-Hallen, "Fading Channel Prediction for Mobile Radio Adaptive Tx. Systems," Proc. of IEEE, vol. 95, no. 12, pp. 2299-2313, 2007.

[10] C. Komninakis, C. Fragouli, A. H. Sayed, R. D. Wesel, "MIMO fading channel tracking and equalization using Kalman estimation," IEEE Trans. on Sign. Proc., vol. 50, pp. 10651076, 2002.

[11] S.H. Huang, T. Jenho, T.C. Yang, S.-W. Cheng, "Model-Based Signal Subspace Channel Tracking for Correlated Underwater
Acoustic Communication Channels," IEEE Journal of Oceanic Engineering, vol.39, no.2, pp.343-356, 2014.

[12] S. Q. Wang, A. Abdi, "Optimal training sequence for efficient MIMO frequency selective fading channel estimation," IEEE Sarnoff Symposium, Mar. 2006.

[13] M. Biguesh, A. B. Gershman, "Training-based MIMO channel estimation: a study of estimator tradeoffs and optimal training signals," IEEE Trans. on Sign. Proc., vol. 54, no.3, pp. 884-893, Mar. 2006.

[14] K. Zhong, X. Lei, S. Li, "Iterative channel estimation and data detection for MIMO-OFDM systems operating in timefrequency dispersive channel under unknown background noise," EURASIP J. on Wireless Comms. Networking, 2013.

[15] L. Toni, A. Conti, "Does Fast Adaptive Modulation Always Outperform Slow Adaptive Modulation?" IEEE Trans. Wireless Commun. vol. 10, no. 5, pp. 150-1513, Mar. 2011.

[16] M. F. Siyau, S.L. Ling, O. Onalaja, M. Ghavami, "MIMO channel estimation and tracking using a novel pilot expansion technique with Paley-Hadamard codes for future generation fast speed communications," The 2nd Int. Conf. Future Generation Communication Technology, London UK, 12-14 Nov. 2013.

[17] J. Seberry and M. Yamada, "Hadamard matrices, sequences and block designs," in D. J. Stinson and J. Dinitz, Eds. Contemporary Design Theory - A Collection of Surveys, John Wiley and Sons, 1992, 431-560.

[18] P. Dent, G. Bottomley and T. Croft, "Jakes fading model revisited," Elec. Letters, vol. 29, no. 13, pp. 1162-1163, 2006.

[19] P. A. Bello, "Characterization of randomly time-variant linear channels," IEEE Trans. Commun. Syst., vol.CS-11, pp.360-393, Dec. 1963.

[20] M. F. Siyau, R. Ormondroyd, P. Nobles, "Performance of a layered ST system with a fractionally-spaced channel estimator in a TS \& FS wireless channels," IEEE on Conf. Veh. Tech., vol. 4, pp. 2467-2472, 2004.

[21] T. E. Bogale, L. B. Le, Pilot Optimization and Channel Estimation for Multiuser Massive MIMO Systems, The 48th Annual Conference in Information Sciences and Systems, March 19-21, 2014, Princeton, NJ, United States.

[22] J. Ma, P. Orlik, J, Zhang; G.Y. Li, "Pilot Matrix Design for Estimating Cascaded Channels in Two-Hop MIMO Amplifyand-Forward Relay Systems," IEEE Transactions on Wireless Communications, vol.10, no.6, pp.1956-1965, 2011.

[23] E. Manasseh, S. Ohno, T. Yamamoto, Efficient training design for estimation of channel, CFO, and receiver I/Q imbalance in MIMO-OFDM systems, in proceedings of 13th International Symposium on Communications and Information Technologies, pp.364-368, 4-6 Sept. 2013.

[24] M. Chiani, A. Conti, C. Fontana, "Improved performance in TD-CDMA mobile radio system by optimizing energy partition in channel estimation," IEEE Transactions on Communications, vol.51, no.3, pp.352-355, 2003.

[25] C. Qi, G. Yue, L. Wu, A. Nallanathan, "Pilot Design for Sparse Channel Estimation in OFDM-Based Cognitive Radio Systems," IEEE Transactions on Vehicular Technology, vol.63, no.2, pp.982-987, 2014.

[26] A. Conti, W.M. Gifford, M.Z. Win, M. Chiani, "Optimized simple bounds for diversity systems," IEEE Transactions on Communications, vol.57, no.9, pp.2674-2685, 2009. 